\title{
ETV6/PDGFRB Fusion Protein
}

National Cancer Institute

\section{Source}

National Cancer Institute. ET V6/PD GFRB Fusion Protein. NCI Thesaurus. Code C99404.

A fusion protein encoded by the ETV6/PDGFRB fusion gene. This protein is comprised of the N-terminal DNA binding helix-loop-helix domain of the transcription factor ETV6 protein fused to the C-terminal transmembrane and cytoplasmic domains of the betatype platelet-derived growth factor receptor protein, including the protein kinase domain. 